\title{
Efficacy and safety of docetaxel and prednisolone chemotherapy in very elderly men with metastatic castration-resistant prostate cancer (mCRPC) in real world: a single institute experience
}

\author{
Kai-Yun Wang ${ }^{1,2}$, Liang Ma ${ }^{1,3}$, Lan-Lan Zhang ${ }^{4}$, Yi-Chao Hu ${ }^{4}$, Jun-Hui Jiang ${ }^{3}$, Qi Ma ${ }^{1,3,4,5}$ \\ ${ }^{1}$ Comprehensive Urogenital Cancer Center, Ningbo First Hospital, The Affiliated Hospital of Ningbo University, Ningbo, China; ${ }^{2}$ Medical School, \\ Ningbo University, Ningbo, Zhejiang, China; ${ }^{3}$ Department of Urology, Ningbo First Hospital, The Affiliated Hospital of Ningbo University, \\ Ningbo, China; ${ }^{4}$ Ningbo Clinical Research Center for Urological Disease, Ningbo, China; ${ }^{5}$ Translational Research Laboratory for Urology, the Key \\ Laboratory of Ningbo City, Ningbo First Hospital, The Affiliated Hospital of Ningbo University, Ningbo, China \\ Contributions: (I) Conception and design: Q Ma, JH Jiang; (II) Administrative support: JH Jiang; (III) Provision of study materials or patients: L \\ Ma, KY Wang, Q Ma; (IV) Collection and assembly of data: YC Hu, LL Zhang, KY Wang; (V) Data analysis and interpretation: KY Wang; (VI) \\ Manuscript writing: All authors; (VII) Final approval of manuscript: All authors. \\ Correspondence to: Dr. Qi Ma. Translational Research Laboratory for Urology, the Key Laboratory of Ningbo City, Ningbo First Hospital, The \\ Affiliated Hospital of Ningbo University, No. 59 Liu-Ting Street, Ningbo 315010, China. Email: qi_ma@yahoo.com.
}

Backgroundk Prostate cancer is the most common type of malignancy in elderly men. Although elderly patients are commonly encountered in clinical practice, few studies have focused on the value of chemotherapy in elderly patients. In this study, we reviewed the use of docetaxel with prednisolone in elderly men (aged $\geq 80$ years) with metastatic castration-resistant prostate cancer (mCRPC) at Ningbo First Hospital with a focus on efficacy and toxicity.

Methods: A retrospective study including a series of men aged $\geq 80$ years with mCRPC and received docetaxel plus prednisone chemotherapy between August 2011 and May 2019. All these cases were selected from the Ningbo First Hospital prostate cancer database located in Zhejiang Province, China.

Results: Sixteen patients were identified, with a mean age of 82 years (range, 80 to 87 years). All patients have received a median of four and half cycles (range, 1-10) of 3-week $\left(60-75 \mathrm{mg} / \mathrm{m}^{2}\right)$ docetaxel regimens and $5 \mathrm{mg}$ prednisone twice per day. Seven (43.75\%) patients completed more than six cycles. Ten $(62.50 \%)$ patients had a good prostate-specific antigen (PSA) response of $\geq 50 \%$ decline. Eight (50.00\%) patients had ostealgia before receiving docetaxel treatment and six of them $(75.00 \%)$ experienced reduced pain after the treatment. Hematologic toxicity was observed in six $(37.50 \%)$ patients with neutropenia, one of which was diagnosed with agranulocytosis and had to be admitted for the same reason. Other adverse reactions such as fever, debilitation, and alopecia were also observed.

Conclusions: Very elderly patients (aged $\geq 80$ years) with mCRPC are easy to be neglected and infrequently involved in clinical trials. Our study demonstrates that docetaxel chemotherapy plus prednisone is tolerable and effective among Chinese elderly patients ( $\geq 80$ years) with mCRPC. Docetaxel chemotherapy may be given under careful surveillance even in frail elderly patients.

Keywords: Very elderly; docetaxel chemotherapy; metastatic castration-resistant prostate cancer (mCRPC); real world

Submitted Mar 06, 2020. Accepted for publication Sep 18, 2020.

doi: 10.21037/apm-20-573a

View this article at: http://dx.doi.org/10.21037/apm-20-573a 


\section{Introduction}

Prostate cancer is the most common type of malignancy in elderly men (1). The incidence of prostate cancer has increased rapidly in China over the last 40 years and there are 47 thousand new cases annually (2-4). Prostate cancer is predominantly a geriatric disease as incidence and mortality increase with age (5).

Androgen deprivation therapy (ADT) is the primary systemic treatment choice for patients with metastatic prostate cancer. ADT induced remissions typically last for 2 to 3 years but most patients will progress to a castrationresistant state despite having an anorchid testosterone level (6). The treatment for patients with metastatic castrationresistant prostate cancer (mCRPC) has evolved significantly in recent years (7). Docetaxel was the first agent to demonstrate the improvement of survival rate in mCRPC patients based on two landmark phase III trials TAX327 and SWOG9916, as well as their subsequent studies (8-10). The combination of docetaxel and steroids (prednisone $5 \mathrm{mg}$ twice daily plus docetaxel $75 \mathrm{mg} / \mathrm{m}^{2}$ every 3 weeks) was approved by the Food and Drug Administration (FDA) in 2004 and became the standard first-line treatment for mCRPC (11). Following that, several new drugs and one therapeutic vaccine were also approved by the FDA for the treatment of mCRPC (12-15). However, most of the new treatments are expensive with a low average cost-effectiveness ratio, rendering them unsuitable for routine clinical care in China.

Although elderly patients are commonly encountered in clinical practice, few studies have focused on the value of chemotherapy in elderly patients $(13,16)$. In elderly patients, the risk of toxicity is a significant concern with chemotherapy. In a subgroup analysis of the TAX327 trial, men aged $\geq 75$ years had significantly higher rates of infection and required more frequent dose reductions than younger patients (17). Elderly patients were also more likely to present with metastatic disease at diagnosis and patients aged $\geq 80$ years accounted for over half of prostate cancer-related deaths (16).

In this retrospective study, we reviewed the use of docetaxel with prednisolone in elderly men (aged $\geq 80$ years) with mCRPC at Ningbo First Hospital with a focus on efficacy and toxicity. We present the following article in accordance with the STROBE reporting checklist (available at http://dx.doi.org/10.21037/apm-20-573a).

\section{Methods}

All procedures performed in this study involving human participants were in accordance with the Declaration of Helsinki (as revised in 2013). The ethics approval of this study was obtained from the ethics committees of Ningbo First Hospital (No. 2019-R023-01). Informed consent was obtained from all patients and their families.

\section{Patients}

Men aged $\geq 80$ years diagnosed with $\mathrm{mCRPC}$ and received docetaxel plus prednisone chemotherapy between August 2011 and May 2019 were selected from the Ningbo First Hospital prostate cancer database located in Zhejiang Province, China. All patients had histologically confirmed metastatic prostate adenocarcinoma despite castration and anti-androgen therapy had failed prior to chemotherapy. All treatment and response data were extracted from the hospitals' record system.

\section{Treatment protocol}

Docetaxel was given as a 3 -week $\left(60-75 \mathrm{mg} / \mathrm{m}^{2}\right)$ regimen prednisone was given $5 \mathrm{mg}$ for twice per day. Treatment with docetaxel was continued until disease progression, unacceptable toxicities, patients' refusal or death. Granulocyte colony-stimulating factor prophylaxis was administered to patients at the discretion of the attending oncologist. Androgen deprivation was continued with luteinizing hormone-releasing hormone agonists if the patient had not undergone a bilateral orchidectomy; $4 \mathrm{mg}$ zoledronic acid was routinely infused post-chemotherapy to patients diagnosed with bone metastasis with normal serum creatinine level.

Post-docetaxel treatment decisions were determined by several factors including but not limited to the patient's clinical condition, physician's preference, and patient's affordability. Most of the patients received abiraterone while the remaining continued with the ADT treatment.

\section{Efficacy and safety assessment}

Patient prostate-specific antigen (PSA) responses, pain control, treatment toxicities, number of cycles received and the overall survival rate were reviewed and analyzed. A PSA response was defined as a decline in the serum level of more than $50 \%$ between pre- and post-treatment. Pain control was derived from the patients' clinical notes after reviewing the amount of analgesic consumed and symptom descriptions. Complete cessation of analgesic and being pain-free was defined as complete resolution of pain and decreased analgesic 
Table 1 Baseline characteristics of sixteen patients with mCRPC who received docetaxel-based treatment

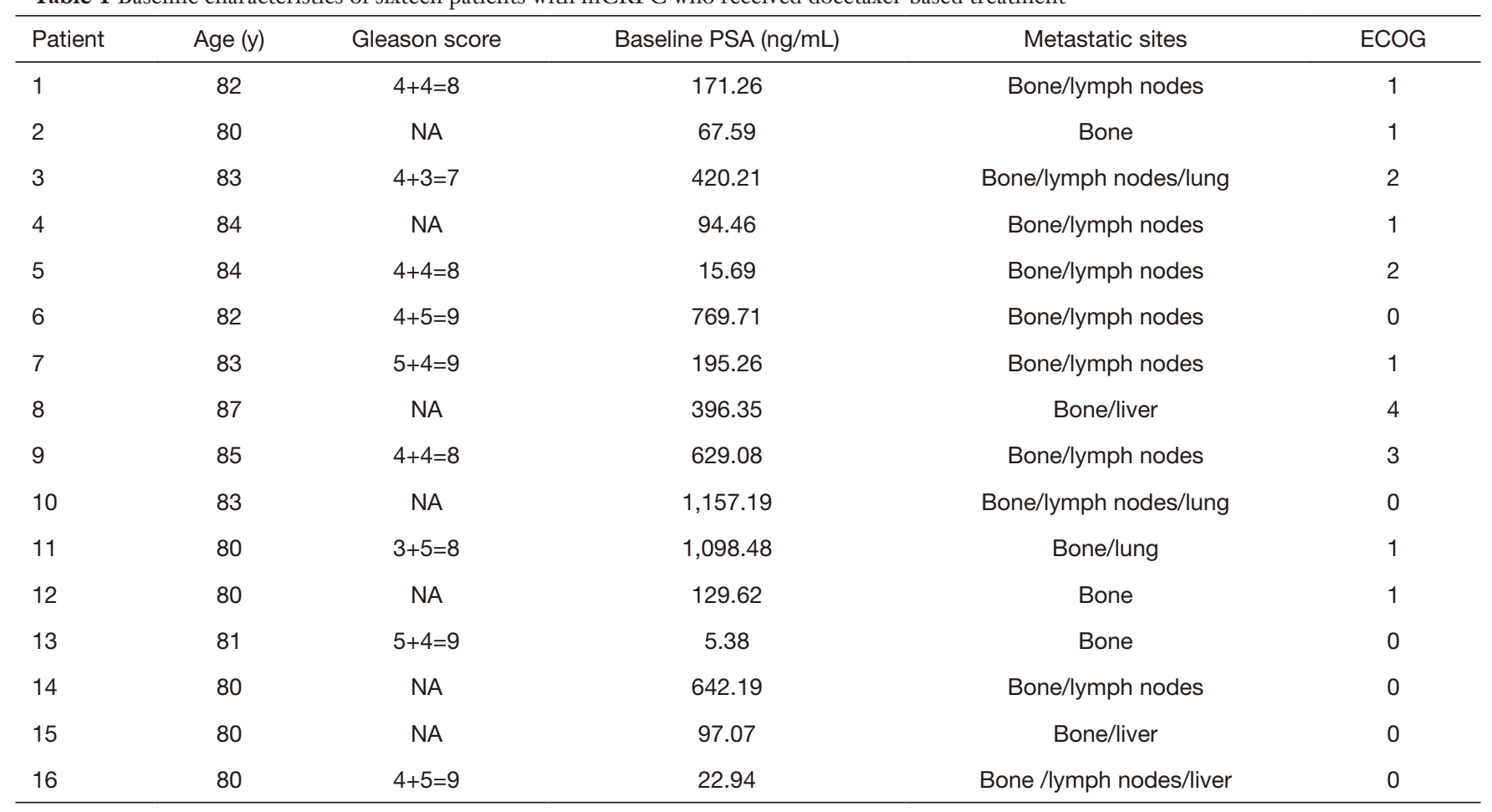

PSA, prostate-specific antigen; ECOG, Eastern Cooperative Oncology Group performance status; N/A, data was not available.

consumption associated with "less pain" was defined as an improvement in pain control. Successful pain control is one of the conditions necessary for an effective docetaxel treatment.

Hematological complications were retrospectively graded using the United States.

Department of Health and Human Services Common Terminology Criteria for Adverse Events version 4.0 (CTCAE). The number of patients who complained of systemic side-effects such as malaise, debilitation and ankle edema were derived from the patients' clinical notes.

\section{Statistical analysis}

Patients' data were summarized and tabulated using descriptive statistics. Overall survival was calculated using the Kaplan-Meier method on the statistical software program SPSS 22.0 (IBM, Armonk NY). Statistical significance was set at $\mathrm{P} \leq 0.05$.

\section{Results}

\section{Patient characteristics}

Sixteen men with a mean age of 82 years (range, 80 to
87 years) were selected. Patients and disease characteristics are presented in Table 1. The Eastern Cooperative Oncology Group (ECOG) score performance status was documented for all patients. Twelve patients had a good to moderate performance status of ECOG 0 to 1 (75.00\%).

Seven $(43.75 \%)$ patients had bone and lymph node metastasis. Three (18.75\%) patients had bone-only diseases, five $(31.25 \%)$ patients had visceral metastases and one $(6.25 \%)$ patient had retroauricular lymphadenopathy. The median pre-treatment serum PSA of all patients was $183.26 \mathrm{ng} / \mathrm{mL}$ (range, $5.38-1,157.19 \mathrm{ng} / \mathrm{mL}$ ). One (6.25\%) patient required daily opioid analgesia due to severe pain.

\section{Treatment delivery}

All patients received a median of four and a half cycles (range, 1-10) of a 3 -week $\left(60-75 \mathrm{mg} / \mathrm{m}^{2}\right)$ regimen of docetaxel treatment and $5 \mathrm{mg}$ prednisone twice per day. Seven $(43.75 \%)$ patients completed more than six cycles. Five $(31.25 \%)$ patients only received one cycle for personal reasons. Two patients discontinued treatment early due to adverse events such as agranulocytosis, fever, and alopecia. One patient died after two cycles of chemotherapy due 


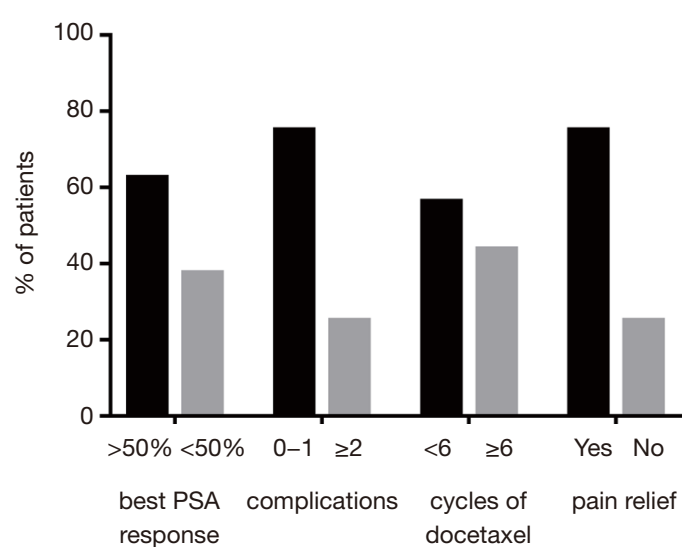

Figure 1 The stratified data of all sixteen patients' best PSA response, post chemotherapy complications, cycles and pain relief of docetaxel-based treatment. PSA, prostate-specific antigen.

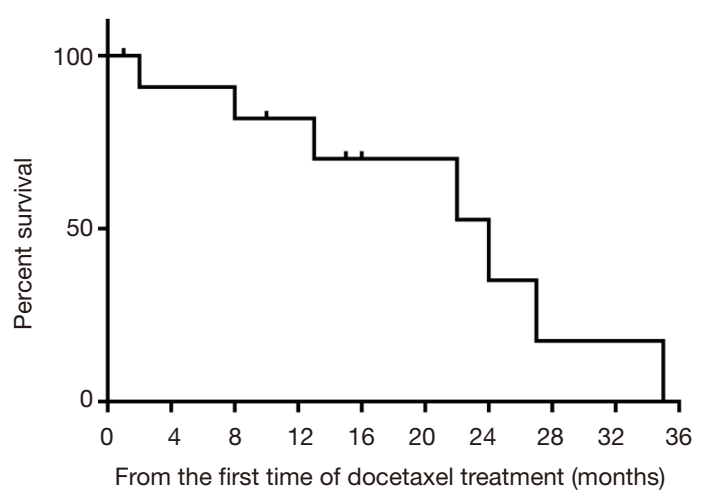

Figure 2 Overall survival (OS) for all sixteen patients with mCRPC. These "I" on the line means the time when several patients were lost to follow-up.

heart disease.

\section{Efficacy}

PSA response was measurable for all sixteen patients. In our study, ten $(62.50 \%)$ patients had a good PSA response of $\geq 50 \%$ decline. Eight $(50.00 \%)$ patients had ostealgia before receiving docetaxel treatment and six $(75.00 \%)$ of those patients experienced reduced pain after the treatment (including one patient who required daily opioid analgesia). A summary of the PSA response, complications, number of docetaxel treatment cycles and pain relief of all sixteen patients is presented in Figure 1.

Follow-up data were recorded up to 17 May 2019. Twelve patients were lost to follow up, one patient had died due to heart disease and three patients were still receiving new hormonal therapy. The patient with retroauricular lymphadenopathy also improved after three cycles of docetaxel. The overall survival is presented in Figure 2.

\section{Toxicities}

Kongsted (18) found that patients of older age or those with a history of metastatic epidural spinal cord compression (MESCC), have a higher risk of chemotherapy-induced toxicity. In this study, hematologic toxicity was observed in six $(37.50 \%)$ patients with neutropenia, one of which was diagnosed with agranulocytosis and had to be admitted for the same reason. Eight (50\%) patients had $\geq 1$ complicated condition(s), the most common being granulocytopenia $(n=5)$ and edema $(n=4)$. Other adverse reactions such as fever, debilitation, and alopecia were also observed.

A summary of the treatment cycles, toxicities, and efficacy of all sixteen patients who received docetaxel treatment is presented in Table 2.

\section{Discussion}

Multiple new agents have been approved for the treatment of $\mathrm{mCRPC}$ in recent years, these new agents include the dendritic cell vaccine (sipuleucel-T), chemotherapy (cabazitaxel), new hormonal therapeutic agents targeting androgen biosynthesis (abiraterone), androgen receptor drug (enzalutamide), radium-223 and the latest checkpoint inhibitor PD1/PDL1. However, no optimal treatment plan for elderly patients with mCRPC exists due to a lack of prospective randomized trial data to guide clinical decisionmaking. New hormonal therapeutic agents are more tolerable for elderly patients due to the lower toxicity rate. However, both abiraterone and enzalutamide are costly and are unaffordable for many patients with mCRPC.

In elderly patients, a significant concern with docetaxelbased chemotherapy is the risk of severe toxicity. Shigeta (19) suggested that men older than 75 years are more prone to adverse events. Therefore, this group of patients requires constant monitoring to prevent toxicity induced adverse events (20). Conversely, Maia (21) found that patients aged 75 years or older developed neutropenia, febrile neutropenia, and anemia less frequently, while the other types of adverse events did not differ significantly among the different age groups.

In our retrospective study, very elderly patients (aged $\geq 80$ years) responded significantly well to docetaxel-based 
Table 2 Treatment cycles, efficacy, and toxicities of sixteen patients with mCRPC who received docetaxel-based treatment

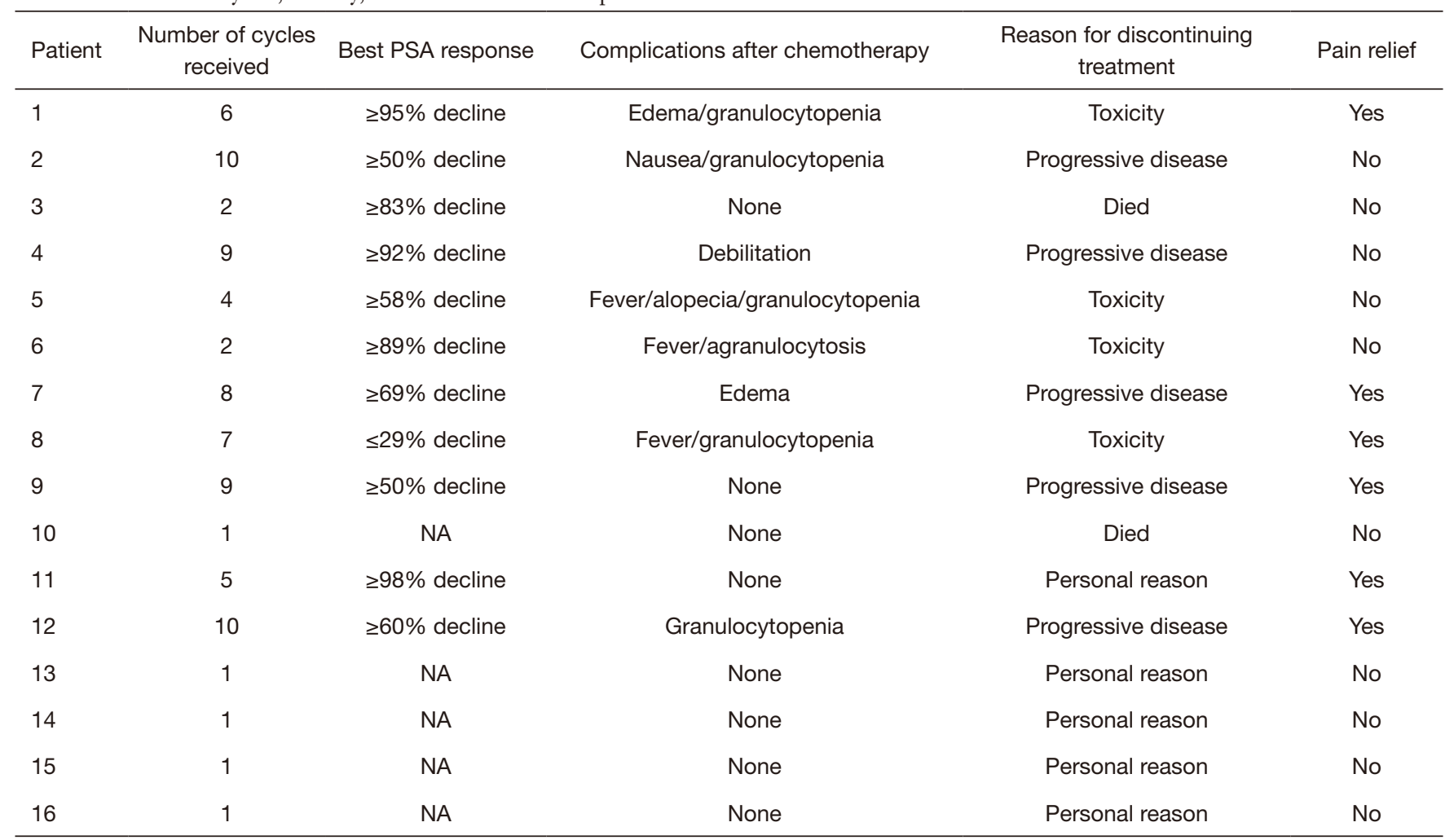

PSA, prostate-specific antigen; mCRPC, metastatic castration-resistant prostate cancer.

chemotherapy. In patients who received at least two cycles of docetaxel treatment, ten $(90.91 \%)$ patients experienced a $>50 \%$ decline in PSA and five patients experienced relief from ostealgia, which includes one patient whose pain required daily opioid analgesia. Surprisingly, there were three patient who had a PSA response of over $90 \%$; one of the three patients who had a baseline PSA of $1,098.48 \mathrm{ng} / \mathrm{mL}$ responded significantly well to docetaxel treatment, reducing his PSA level to $12.50 \mathrm{ng} / \mathrm{mL}$ from just one cycle and his PSA was $0.03 \mathrm{ng} / \mathrm{mL}$ by the fifth cycle of docetaxel treatment. Though docetaxel-based chemotherapy was effective in this series, adverse events such as agranulocytosis, fever, edema, debilitation, and alopecia were observed. The main toxicity observed in our study was leukocytosis. Five patients received neutropenia treatment and continued with the docetaxel chemotherapy, but one patient with agranulocytosis quit and received new hormonal therapy.

Five patients received only one cycle of docetaxel treatment before quitting due to personal reasons such as financial or cultural reasons. Some Chinese perceive chemotherapy as 'demons' and believe that only dying patients accept chemotherapy. There is still a long way to go for urologists in China to get patients to accept chemotherapy as a general treatment.

There are several limitations to this study. First, as with all retrospective studies, the data for some patients is incomplete. Second, patient selection bias due to the small sample size. Third, this is a single-center study. Nevertheless, the study achieved its aims and showed that the docetaxel plus prednisone chemotherapy was effective and well-tolerated in elderly mCRPC patients. Although this small study highlights the value of docetaxel plus prednisone chemotherapy in treating elderly patients with mCRPC routinely, prospective multicenter studies are still necessary.

In conclusion, our study showed that docetaxel chemotherapy with $5 \mathrm{mg}$ prednisone twice daily is tolerable and effective in Chinese elderly patients ( $\geq 80$ years). Even in frail elderly patients, docetaxel chemotherapy may be given under careful surveillance. A comprehensive geriatric assessment is helpful for the identification of patients who 
are at risk of increased toxicity.

\section{Acknowledgments}

The assistance from Dr. Derry Minyao Ng and Dr. Maria Haleem (School of Medicine, Ningbo University, Ningbo, China) in editing the manuscript was greatly appreciated. Funding: This study was supported by Zhejiang Natural Science Fund (Grant No. LY20H050002 to Q Ma, Grant No. LY18H05000 to JH Jiang). Ningbo Natural Science Foundation (Grant No. 2018A610297 to Q Ma), Zhejiang TCM Science and Technology Program (Grant No. 2020ZB217 to Q Ma), and the Fund of Ningbo Clinical Research Center for Urological Disease (2019A21001).

\section{Footnote}

Reporting Checklist: The authors have completed the STROBE reporting checklist. Available at http://dx.doi. org/10.21037/apm-20-573a

Data Sharing Statement: Available at http://dx.doi. org/10.21037/apm-20-573a

Conflicts of Interest: All authors have completed the ICMJE uniform disclosure form (available at http://dx.doi. org/10.21037/apm-20-573a). The authors have no conflicts of interest to declare.

Ethical Statement: The authors are accountable for all aspects of the work in ensuring that questions related to the accuracy or integrity of any part of the work are appropriately investigated and resolved. All procedures performed in this study involving human participants were in accordance with the Declaration of Helsinki (as revised in 2013). The ethics approval of this study was obtained from the ethics committees of Ningbo First Hospital (No. 2019R023-01). Informed consent was obtained from all patients and their families. Identifying or sensitive information was not presented.

Open Access Statement: This is an Open Access article distributed in accordance with the Creative Commons Attribution-NonCommercial-NoDerivs 4.0 International License (CC BY-NC-ND 4.0), which permits the noncommercial replication and distribution of the article with the strict proviso that no changes or edits are made and the original work is properly cited (including links to both the formal publication through the relevant DOI and the license). See: https://creativecommons.org/licenses/by-nc-nd/4.0/.

\section{References}

1. Siegel RL, Miller KD, Jemal A. Cancer statistics, 2019. CA Cancer J Clin 2019;69:7-34.

2. Chen $\mathrm{W}$, Zheng R, Zeng H, et al. Annual report on status of cancer in China, 2011. Chin J Cancer Res 2015;27:2-12.

3. Torre LA, Bray F, Siegel RL, et al. Global cancer statistics, 2012. CA Cancer J Clin 2015;65:87-108.

4. Kimura T. East meets West: ethnic differences in prostate cancer epidemiology between East Asians and Caucasians. Chin J Cancer 2012;31:421-9.

5. Droz JP, Balducci L, Bolla M, et al. Management of prostate cancer in older men: recommendations of a working group of the International Society of Geriatric Oncology. BJU Int 2010;106:462-9.

6. Garcia JA, Hutson TE, Dale S, et al. Gemcitabine and docetaxel in metastatic, castrate-resistant prostate cancer: results from a phase 2 trial. Cancer 2011;117:752-7.

7. Mogensen SW, Petersen AH, Buchardt AS, et al. Survival prognosis and variable selection: A case study for metastatic castrate resistant prostate cancer patients. F1000Res 2016;5:2680.

8. de Bono JS, Smith MR, Saad F, et al. Subsequent Chemotherapy and Treatment Patterns After Abiraterone Acetate in Patients with Metastatic Castration-resistant Prostate Cancer: Post Hoc Analysis of COU-AA-302. Eur Urol 2017;71:656-64.

9. Mottet N, Bellmunt J, Bolla M, et al. EAU-ESTROSIOG Guidelines on Prostate Cancer. Part 1: Screening, Diagnosis, and Local Treatment with Curative Intent. Eur Urol 2017;71:618.

10. Petrioli R, Francini E, Roviello G. Is there still a place for docetaxel rechallenge in prostate cancer? World J Clin Oncol 2015;6:99-103.

11. Schweizer MT, Antonarakis ES. Chemotherapy and its evolving role in the management of advanced prostate cancer. Asian J Androl 2014;16:334-40.

12. Lee JL, Ahn JH, Choi MK, et al. Gemcitabine-oxaliplatin plus prednisolone is active in patients with castrationresistant prostate cancer for whom docetaxel-based chemotherapy failed. Br J Cancer 2014;110:2472-8.

13. Sternberg CN, Bono JS, De, Chi KN, et al. Improved outcomes in elderly patients with metastatic castrationresistant prostate cancer treated with the androgen receptor inhibitor enzalutamide: results from the phase III 
AFFIRM trial. Ann Oncol 2014;25:429-34.

14. Aparicio AM, Harzstark AL, Corn PG, et al. Platinumbased chemotherapy for variant castrate-resistant prostate cancer. Clin Cancer Res 2013;19:3621-30.

15. Aapro MS. Management of advanced prostate cancer in senior adults: the new landscape. Oncologist 2012;17 Suppl 1:16-22.

16. Wong HL, Lok SW, Wong S, et al. Docetaxel in very elderly men with metastatic castration-resistant prostate cancer. Prostate Int 2015;3:42-6.

17. Antonarakis ES, Tagawa ST, Galletti G, et al. Randomized, Noncomparative, Phase II Trial of Early Switch From Docetaxel to Cabazitaxel or Vice Versa, With Integrated Biomarker Analysis, in Men With Chemotherapy-Naïve, Metastatic, Castration-Resistant Prostate Cancer. J Clin Oncol 2017;35:3181-8.

Cite this article as: Wang KY, Ma L, Zhang LL, Hu YC, Jiang JH, Ma Q. Efficacy and safety of docetaxel and prednisolone chemotherapy in very elderly men with metastatic castrationresistant prostate cancer (mCRPC) in real world: a single institute experience. Ann Palliat Med 2021;10(2):1438-1444. doi: 10.21037/apm-20-573a
18. Kongsted P, Svane IM, Lindberg H, et al. Predictors of Chemotherapy-Induced Toxicity and Treatment Outcomes in Elderly Versus Younger Patients With Metastatic Castration-Resistant Prostate Cancer. Clin Genitourin Cancer 2016;14:e559-e568.

19. Shigeta K, Kosaka T, Yazawa S, et al. Predictive factors for severe and febrile neutropenia during docetaxel chemotherapy for castration-resistant prostate cancer. Int J Clin Oncol 2015;20:605-12 .

20. Wildiers H, Heeren P, Puts M, et al. International Society of Geriatric Oncology consensus on geriatric assessment in older patients with cancer. J Clin Oncol 2014;32:2595-603.

21. Maia MC, Pereira AAL, Lage LV, et al. Efficacy and Safety of Docetaxel in Elderly Patients With Metastatic Castration-Resistant Prostate Cancer. J Glob Oncol 2018;4:1-9. 